\title{
MEROMORPHIC STARLIKE MULTIVALENT FUNCTIONS $\left({ }^{1}\right)$
}

BY

W. C. ROYSTER

1. Introduction. In recent years there has been considerable interest in classes of functions meromorphic in the unit circle with a simple pole at the origin. For instance, it was shown by Clunie [2] that if $f(z)=z^{-1}+a_{0}+a_{1} z+\cdots$ is meromorphic starlike and univalent in $|z|<1$ then $\left|a_{n}\right| \leqq 2(n+1)^{-1}$ (actually Clunie considers the case where $a_{0}=0$; however, it has been remarked in several places in the literature that his proof holds whenever $a_{0} \neq 0$ ). Similar results have been found for close-to-convex meromorphic functions by Libera and Robertson [6] and Pommerenke [8]. Hence to a certain extent, the work in the meromorphic univalent case has paralleled the regular case.

For the class of multivalent functions very little has been done in the meromorphic case (see Biernacki [1]) to study the theory analogous to the regular multivalent theory. Since the regular multivalent theory has many problems left unsolved that have a solved counterpart in the regular univalent theory (see [4]) it is to be expected that the class of meromorphic multivalent functions will afford greater resistance to the efforts to solve these problems.

We plan to study some coefficient problems pertaining to meromorphic multivalently starlike functions of order $p$.

Definition 1. A function $f(z)$ meromorphic in the unit circle is said to be multivalently starlike of order $p$ (or $p$-valently starlike) in $|z|<1$ if there exists a positive number $\rho, 0<\rho<1$, such that the circles $|z|=r, \rho<r<1$ are mapped by $f$ onto curves starlike with respect to the origin, that is, curves having the property that the vector joining a point on the curve with the origin is continuously turning through an angle of $2 p \pi$. Analytically, $f$ satisfies the conditions

$$
\begin{aligned}
\operatorname{Re}\left\{\frac{z f^{\prime}(z)}{f(z)}\right\} & \leqq 0 \\
\int_{0}^{2 \pi} \operatorname{Re}\left\{\frac{z f^{\prime}(z)}{f(z)}\right\} d \theta & =-2 \pi p, \quad z=r e^{i \theta}, \quad \rho<r<1 .
\end{aligned}
$$

For regular starlike multivalent functions of order $p$ Robertson [9] has obtained the sharp bounds on the coefficients in the Taylor expansion provided that $f$ has a zero of order at least equal to $p-1$ at the origin.

Received by the editors May 3, 1962.

(1) Research supported by the National Science Foundation. 
Let $\Sigma_{p}$ be the class of functions

$$
f(z)=\frac{1}{z^{p}}+\frac{a_{-p+1}}{z^{p-1}}+\cdots+a_{n} z^{n}+\cdots
$$

which are regular, except for a pole of order $p$ at the origin, and $p$-valent in $|z|<1$. We shall be concerned with the subclass $\Sigma_{p}^{*}$ of functions belonging to $\Sigma_{p}$ which are starlike with respect to the origin and with an associated class $\Sigma_{p}^{*}(\alpha)$ of functions which are $p$-valently starlike and meromorphic in $|z|<1$ and have a pole at $z=\alpha,|\alpha|<1$. Among other things, we obtain sharp bounds on the first $p$ coefficients in the expansion of $f \in \Sigma_{p}^{*}$ and show that $\left|a_{n}\right|=O(1 / n)$ for large $n$. By taking the coefficients $a_{-p+1}=\cdots=a_{-1}=0$ we are able to obtain the sharp bound for all of the other $\left|a_{n}\right|$. For $\Sigma_{p}^{*}(\alpha)$ we obtain the sharp bound on the second coefficient. Finally, we obtain the radius of starlikeness for $\Sigma_{p}$.

2. Coefficient bounds. Let $f(z)=R e^{i \Phi}$ belong to $\Sigma_{p}$ then Golusin [3] and Kobori [5] have shown that $\int_{L_{r}} R^{\lambda} d \Phi \leqq 0, \lambda>0$, where $L_{r}$ is the map of $|z|=r<1$. Since

we have

$$
\int_{L_{r}} R^{\lambda} d \Phi=\frac{r}{\lambda} \frac{\partial}{\partial r} \int_{0}^{2 \pi} R^{\lambda} d \theta
$$

$$
\frac{\partial}{\partial r} \int_{0}^{2 \pi}\left|f\left(r e^{i \theta}\right)\right|^{\lambda} d \theta \leqq 0 .
$$

Upon setting $\lambda=2$ and substituting into these integrals we get the area theorems of Golusin and Kobori, that is,

$$
\sum_{n=p+1}^{\infty}(n-p)\left|a_{n-p}\right|^{2} \leqq p+(p-1)\left|a_{-p+1}\right|^{2}+\cdots+\left|a_{-1}\right|^{2} .
$$

In addition to the area principle, and indeed by employing it, Golusin proved that if $f \in \Sigma_{p}$ and is never zero in $|z|<1$ then $\left|a_{-p+1}\right| \leqq 2 p$ and $\left|a_{-p+2}\right| \leqq p(2 p-1)$. Both bounds are sharp.

We extend these bounds to higher ordered coefficients for the class $\Sigma_{p}^{*}$.

TheOREM 1. Let $f(z)=z^{-p}+a_{-p+1} z^{-p+1}+\cdots$ belong to $\Sigma_{p}^{*}$; then

$$
\left|a_{-p+k}\right| \leqq\left(\begin{array}{c}
2 p \\
k
\end{array}\right) \text { for } k=1,2, \cdots, p .
$$

The inequality (3) is sharp as is shown by the function $f_{0}(z)=z^{-p}\left(1+e^{i \beta} z\right)^{2 p}$, $\beta$ real.

Proof. A method due to Clunie generalizes to the $p$-valent case. Let $h(z)$ be a function regular in $|z|<1$ and satisfying the properties $h(0)=0$ and 
$|h(z)| \leqq 1$. By hypothesis $\operatorname{Re}\left\{z f^{\prime} / f\right\}<0$ for $0<\rho<r<1$. Since $z f^{\prime} / f$ is regular in $|z|<1$, we can write $z f^{\prime} / f=-p\left(1+c_{1} z+c_{2} z^{2}+\cdots\right)=-p g(z)$ where $\operatorname{Re}\{g(z)\} \geqq 0$ in $|z|<1$ and $g(0)=1$. Now we may write $h(z)=(g(z)-1)(g(z)+1)^{-1}$ which when written in terms of $f$ yields

$$
p f(z)+z f^{\prime}(z)=h(z)\left[p f(z)-z f^{\prime}(z)\right] .
$$

When expanded in series (4) becomes

whence

$$
\sum_{k=-p+1}^{\infty}(k+p) a_{k} z^{k}=\left(\sum_{k=1}^{\infty} h_{k} z^{k}\right)\left(2 p z^{-p}+\sum_{k=-p+1}^{\infty}(p-k) a_{k} z^{k}\right)
$$

$$
\sum_{k=-p+1}^{n}(k+p) a_{k} z^{k}+\sum_{k=n+1}^{\infty} b_{k} z^{k}=h(z)\left(2 p z^{-p}+\sum_{k=-p+1}^{n-1}(p-k) a_{k} z^{k}\right) .
$$

If we multiply each side of (6) by its conjugate and integrate around $|z|=r<1$ and use the fact that $|h(z)| \leqq 1$ we get, letting $r \rightarrow 1$,

$$
\sum_{k=-p+1}^{n}(k+p)^{2}\left|a_{k}\right|^{2} \leqq 4 p^{2}+\sum_{k=-p+1}^{n-1}(p-k)^{2}\left|a_{k}\right|^{2}
$$

which yields after some manipulation

$$
(n+p)^{2}\left|a_{n}\right|^{2} \leqq 4 p\left[p+(p-1)\left|a_{-p+1}\right|^{2}+\cdots+\left|a_{-1}\right|^{2}-\sum_{k=1}^{n-1} k\left|a_{k}\right|^{2}\right]
$$

or

$$
m^{2}\left|a_{-p+m}\right|^{2} \leqq 4 p\left[p+(p-1)\left|a_{-p+1}\right|^{2}+\cdots+\left|a_{-1}\right|^{2}-\sum_{k=1}^{m-p-1} k\left|a_{k}\right|^{2}\right] .
$$

For $m=1$, we have $\left|a_{-p+1}\right| \leqq 2 p ; m=2,\left|a_{-p+2}\right| \leqq p(2 p-1)=C_{2 p, 2}$. We easily establish that

$$
4 p\left[p+(p-1)\left(\begin{array}{c}
2 p \\
1
\end{array}\right)^{2}+\cdots+(p-k+1)\left(\begin{array}{c}
2 p \\
k-1
\end{array}\right)^{2}\right]=k^{2}\left(\begin{array}{c}
2 p \\
k
\end{array}\right)^{2}
$$

for $0 \leqq k \leqq p$. From this identity it follows that

$$
(k+1)^{2}\left|a_{-p+k+1}\right|^{2} \leqq(2 p-k)^{2}\left(\begin{array}{c}
2 p \\
k
\end{array}\right)^{2}, k+1 \leqq p
$$

hence $\left|a_{-p+k+1}\right| \leqq C_{2 p, k+1}, 0<k+1 \leqq p$ which proves the theorem. In the divalent case this same procedure yields $\left|a_{-2+k}\right| \leqq C_{4, k}$ for $k=1,2,3$, that is, the inequality (3) holds for $k=p+1$.

Although the above method does not yield the sharp bounds for $\left|a_{n}\right|, n \geqq 1$, $p \neq 2$, it does give the order of magnitude of the coefficients as $n$ becomes large. Indeed, we obtain from (8) 


$$
(n+p)^{2}\left|a_{n}\right|^{2} \leqq 4 p^{2}+4 p \sum_{k=0}^{p-1} k\left|a_{-k}\right|^{2} \leqq p^{2}\left(\begin{array}{c}
2 p \\
p
\end{array}\right)^{2}
$$

hence

$$
\left|a_{n}\right| \leqq \frac{p\left(\begin{array}{c}
2 p \\
p
\end{array}\right)}{n+p}=O\left(\frac{1}{n}\right) \text { as } n \rightarrow \infty .
$$

THEOREM 2. Let $f \in \Sigma_{p}^{*}$ then $\left|a_{n}\right|=O(1 / n)$. The function $f(z)=z^{-p}\left(1+z^{n}\right)^{2 p / n}$ shows that the order cannot be improved.

Golusin [3] has shown also that if $f \in \Sigma_{p}$ and satisfies (a) $f(z) \neq 0,|z|<1$, (b) $a_{-p+1}=a_{-p+2}=\cdots=a_{-p+k-1}=0, k \geqq 1$, then $\left|a_{n}\right| \leqq 2 p / n$ for $n=k$, $k+1, \cdots, 2 k-1$. In the following theorem we shall show that if $a_{-p+1}=a_{-p+2}$ $=\cdots=a_{-1}=0$ and $f \in \Sigma_{p}^{*}$ then sharp bounds can be obtained for all $n$.

THEOREM 3. Let $f(z)=z^{-p}+a_{0}+a_{1} z+\cdots$ belong to $\Sigma_{p}^{*}$ then

$$
\left|a_{n}\right| \leqq(2 p) /(n+p) \text { for all } n \geqq 0 \text {. }
$$

Equality holds for the function $f_{0}(z)=z^{-p}\left(1+z^{n+p}\right)^{2 p /(n+p)}$.

Proof. Under the assumptions made in the theorem (8) becomes

$$
(n+p)^{2}\left|a_{n}\right|^{2} \leqq 4 p^{2} .
$$

This establishes the theorem. It is easy to verify that $f_{0}(z)$ belongs to $\Sigma_{p}^{*}$. The zeros of $f_{0}^{\prime}$ lie at the $(n+p)$ roots of unity ( $p$ fixed) and the zeros of $f_{0}$ lie at the $(n+p)$ roots of -1 . Hence, for a particular value of $n, f_{0}$ maps the unit circle onto a $p$-sheeted surface furnished with $(n+p)$ slits of length $2^{2 p /(n+p)}$ making an angle of $2 \pi p /(n+p)$ with one another.

If $f \in \Sigma_{p}^{*}$ the problem $\left|a_{n}\right|=\max , n \geqq 1$, is unsolved provided the coefficients $a_{-p+1}, \cdots, a_{0}$ are not all zero. However, the Pick-Nevanlinna theory as employed by Nehari and Netanyahu [7] yields that if $f \in \Sigma_{p}^{*}$ is extremal for $\left|a_{n}\right|=\max$ then

$$
f(z)=z^{-p} \prod_{j=1}^{n+p}\left(1-\eta_{k} z\right)^{\mu_{j}}, \quad\left|\eta_{k}\right|=1, \quad \mu_{j} \geqq 0, \quad \sum_{j=1}^{n+p} \mu_{j}=2 p .
$$

In the previous paragraphs we have concerned ourselves with functions having a pole of order $p$ at the origin. Let us now suppose that $f$ has poles elsewhere. More precisely, let $f$ have a simple pole at $z=\alpha,|\alpha|<1$ and a pole of order $p-1$ at the origin. Then $f$ has in the neighborhood of the origin the expansion

$$
f(z)=a_{-p+1} z^{-p+1}+a_{-p+2} z^{-p+2}+\cdots .
$$

The function $g(z)=z^{-1}(z-\alpha)(1-\bar{\alpha} z) f(z)$ has all of its poles at the origin. It is easy to show that $\operatorname{Re}\left\{z g^{\prime} / g\right\} \leqq 0$ on $|z|=1$ and that

$$
\int_{0}^{2 \pi} \operatorname{Re}\left\{z g^{\prime} / g\right\} d \theta=-2 p \pi, z=r e^{i \theta}, \rho<r<1 .
$$


Hence $g(z)$ is meromorphically starlike and $p$-valent in $|z|<1$. A simple computation yields

$$
g(z)=-\alpha a_{-p+1} z^{-p}+\left[\left(1+|\alpha|^{2}\right) a_{-p+1}-\alpha a_{-p+2}\right] z^{-p+1}+\cdots .
$$

Theorem 1 now yields, after a trivial transformation,

$$
\left|a_{-p+2}\right| \leqq\left(2 p+\frac{1+|\alpha|^{2}}{|\alpha|}\right)\left|a_{-p+1}\right|
$$

We now have the following:

THEOREM 4. Let $f(z)$ be given by (12) and belong to $\Sigma_{p}^{*}(\alpha)$ then inequality (14) holds. Equality holds essentially for the function

$$
f_{0}(z)=z^{-p+1}(z-\alpha)^{-1}(1-\bar{\alpha} z)^{-1}(1+z)^{2 p} .
$$

The function $f_{0}(z)$ maps $|z|<1$ onto a $p$-sheeted Riemann surface which covers the point at infinity $p$ times and is slit from the origin along a straight line to the image of $\theta=\operatorname{arc} \cos \left[2 p-\left(\alpha+\alpha^{-1}\right)\right] /[2(p-1)]$, where we have assumed without loss of generality that $\alpha$ is real.

In the above case we can generalize to functions $p$-valently starlike in $0<|z|<1$ and of the form $f(z)=a_{-q^{-q}}+a_{-q+1} z^{-q+1}+\cdots, 1 \leqq q<p$ and where $f$ has $p-q$ poles in $|z|<1$ other than at the origin, a pole of order $k$ is counted $k$ times. Indeed, the function

$$
g(z)=z^{-p+q} \prod_{k=1}^{p-q}\left(z-\alpha_{k}\right)\left(1-\bar{\alpha}_{k} z\right) f(z), \quad f\left(\alpha_{k}\right)=\infty,
$$

is $p$-valently starlike and meromorphic in $|z|<1$ and has all of its poles at the origin. A short computation yields

$$
g(z)=A_{p-q} a_{-q} z^{-p}+\left(A_{p-q} a_{-q+1}+B_{p-q} a_{-q}\right) z^{-p+1}+\cdots
$$

where

$$
A_{p-q}=(-1)^{p-q} \prod_{k=1}^{p-q} \alpha_{k}, \quad B_{p-q}=(-1)^{p-q-1} \sum_{j=1, k \neq j}^{p-q} \prod_{k=1}^{p-q} \alpha_{k}\left(1+\left|\alpha_{j}\right|^{2}\right),
$$

which yields, by Theorem 1 , that

$$
\left|a_{-q+1}\right| \leqq\left(2 p+\left|\frac{B_{p-q}}{A_{p-q}}\right|\right)\left|a_{-q}\right|
$$

Inequality (17) reduces to (14) for $q=p-1$.

If we consider the meromorphic functions discussed in Theorem 3 we can, in a certain sense, obtain bounds on the coefficients which are independent of the position of the pole. For, let $f(z)=a_{-p+1} z^{-p+1}+a_{1} z+\cdots$ be meromorphic and starlike of order $p$ in $|z|<1$ and suppose $f$ possesses a simple pole at 
$z=\alpha,|\alpha|<1$, then, again, $g(z)=z^{-1}(z-\alpha)(1-\bar{\alpha} z) f(z)$ has all of its poles at $z=0$ and is $p$-valently starlike in $|z|<1$. We find easily that

$$
\frac{z g^{\prime}(z)}{g(z)}=\gamma_{0}+\gamma_{1} z+\gamma_{2} z^{2}+\cdots
$$

where

$$
\begin{aligned}
& \gamma_{0}=-p, \gamma_{1}=-\left(\bar{\alpha}+\alpha^{-1}\right), \quad \gamma_{2}=-\left(\bar{\alpha}^{2}+\alpha^{-2}\right), \cdots, \\
& \gamma_{p}=p\left(\frac{a_{1}}{a_{-p+1}}-\bar{\alpha}^{p}-\alpha^{-p}\right), \cdots .
\end{aligned}
$$

From Caratheodory's theorem we see immediately that $\left|\bar{\alpha}+\alpha^{-1}\right| \leqq|\alpha|+|\alpha|^{-1}$ $\leqq 2 p$ which establishes a lower bound for $|\alpha|$, in fact, $|\alpha| \geqq p-\left(p^{2}-1\right)^{1 / 2}$. Since $f$ has a simple pole at $z=\alpha$ the function $z f^{\prime} / f$ has a simple pole at $z=\alpha$. Hence the function

$$
h(z)=\frac{(z-\alpha)(1-\bar{\alpha} z)}{z} \frac{z f^{\prime}(z)}{f(z)}
$$

has only a simple pole at $z=0$ with residue $(p-1) \alpha$. The function $F(z)=h(z)-(p-1)\left(\alpha z^{-1}-\bar{\alpha} z\right)$ is then regular in $|z|<1$ and $\operatorname{Re}\left\{F\left(e^{i \theta}\right)\right\} \leqq 0$, hence by the maximum principle $\operatorname{Re}\{F(z)\} \leqq 0$ for $|z|<1$. The function $F$ has the expansion

$$
\begin{aligned}
F(z)= & F(0)+(p-1) \sum_{n=1}^{\infty} c_{n} z^{n} \\
& F(0)=-(p-1)\left(1+|\alpha|^{2}\right) .
\end{aligned}
$$

Upon computing $z f^{\prime} / f$ we find

$$
\begin{aligned}
\frac{z f^{\prime}(z)}{f(z)} & =\frac{(p-1) z}{(z-\alpha)(1-\bar{\alpha} z)}\left[\frac{\alpha}{z}-\left(1+|\alpha|^{2}\right)+\bar{\alpha} z-2 \bar{\alpha} z+c_{1} z+\cdots\right] \\
& =-(p-1)+(p-1) \frac{z}{(z-\alpha)(1-\bar{\alpha} z)}\left[\left(-2 \bar{\alpha}+c_{1}\right) z+\sum_{n=2}^{\infty} c_{n} z^{n}\right] \\
& =-(p-1)+(p-1) G(z) .
\end{aligned}
$$

The function $z f^{\prime} / f$ is regular in $|z|<|\alpha|$, therefore, upon integrating from zero to $z,|z|<|\alpha|$, we get

$$
\frac{z^{p-1} f(z)}{a_{-p+1}}=\exp \left[(p-1) \int_{0}^{z} \frac{G(z)}{z} d z\right] .
$$

If we denote $G(z)=\sum_{n=2}^{\infty} b_{n} z^{n}$, then $b_{n}=b_{n}\left(c_{1}^{\prime}, c_{2}, \cdots, c_{n-1}\right)$ where $c_{1}^{\prime}=c_{1}-2 \bar{\alpha}$, is a linear combination of the complex constants $c_{k}, 1 \leqq k \leqq n-1$. We can now write 


$$
\begin{aligned}
& \exp \left[(p-1) \int_{0}^{z} \frac{G(z)}{z} d z\right] \\
& =1+d_{1} z+d_{2} z^{2}+\cdots=1+\frac{a_{1}}{a_{-p+1}} z^{p}+\frac{a_{2}}{a_{-p+1}} z^{p+1}+\cdots
\end{aligned}
$$

where $d_{n}=d_{n}\left(c_{1}^{\prime}, c_{2}, \cdots, c_{n-1}\right)$ is a homogeneous polynomial in $c_{k}$ with positive coefficients and linear in $c_{n-1}$. Equating coefficients in (23) we have $c_{1}^{\prime}=d_{1}=0, d_{2}=\cdots=d_{p-1}=0$ which implies that $c_{2}=c_{3}=\cdots=c_{p-2}=0$ and $\left(a_{1} / a_{-p+1}\right)=d_{p}=\left[-(p-1) c_{p-1} / p \alpha\right]$. However $\left|(p-1) c_{p-1}\right| \leqq 2|F(0)|$ $=2(p-1)\left(1+|\alpha|^{2}\right)$, hence

$$
\left|a_{1}\right| \leqq \frac{(p-1) \cdot 2\left(1+|\alpha|^{2}\right)}{p|\alpha|}\left|a_{-p+1}\right|=\frac{2(p-1)}{p}\left(|\alpha|+|\alpha|^{-1}\right)\left|a_{-p+1}\right| .
$$

It was shown above that $|\alpha|+|\alpha|^{-1} \leqq 2 p$ which gives the bound

$$
\left|a_{1}\right| \leqq 4(p-1)\left|a_{-p+1}\right| \text {, independent of } \alpha \text {. }
$$

We also note that $\left(a_{2} / a_{-p+1}\right)=\alpha^{-1}(p+1)^{-1}\left[c_{p}+\left(\bar{\alpha}+\alpha^{-1}\right) c_{p-1}\right]$ which yields the bound

$$
\left|a_{2}\right| \leqq 2 \frac{(p-1)}{p+1}\left(|\alpha|+|\alpha|^{-1}\right)\left(1+|\alpha|+|\alpha|^{-1}\right)\left|a_{-p+1}\right|
$$

We have the following

THEOREM 5. Let $f(z)=a_{-p+1} z^{-p+1}+a_{1} z+\cdots$ be meromorphic and p-valently starlike in $|z|<1$ then the inequalities (24), (25) and (26) hold.

Simply by retracing the procedure in the proof of the theorem we can show that (24) and (25) are sharp. Let us suppose for simplicity that $a_{-p+1}=1$ and $\alpha$ is real and positive. Since $c_{1}^{\prime}=c_{2}=\cdots=c_{p-2}=0$ let

$$
\begin{aligned}
h(z) & =(p-1)\left[\alpha z^{-1}-\left(1+\alpha^{2}\right)+\alpha z-2\left(1+\alpha^{2}\right)\left(z^{p-1}+z^{2 p-1}+\cdots\right)\right] \\
& =\alpha(p-1)\left[z^{-1}-2\left(\alpha+\alpha^{-1}\right)+z+\left(\alpha^{-1}+\alpha\right)\left(1-2 z^{p-1}+2 z^{2 p-1}+\cdots\right)\right] .
\end{aligned}
$$

Then

$$
\frac{z f^{\prime}(z)}{f(z)}=\frac{\alpha(p-1) z}{(z-\alpha)(1-\alpha z)}\left[z^{-1}-2\left(\alpha^{-1}+\alpha\right)+z+\left(\alpha^{-1}+\alpha\right) \frac{1-z^{p-1}}{1+z^{p-1}}\right]
$$

Whereupon integrating gives

$$
f(z)=z^{-p+1} \exp \int_{0}^{z} \frac{G(z)}{z} d z
$$

where

$$
G(z)=\frac{2(p-1)\left(\alpha+\alpha^{-1}\right)}{\left(1-\alpha^{-1}\right) z(1-\alpha z)} \frac{z^{p}}{1+z^{p}} .
$$


The function $f$ can be shown to satisfy conditions (a) and (b) of Definition 1 and hence is extremal for (24) and (25), whenever $\alpha=p-\left(p^{2}-1\right)^{1 / 2}$.

It should be mentioned that if $f(z)=a_{-q} z^{-q}+a_{-q+1} z^{-q+1}+\cdots, q$ integral and $1<q<p$, is meromorphic and $p$-valently starlike in $|z|<1$ and does not assume the value infinity more than $q$ times then the methods of Theorems 1 and 2 will yield bounds on the coefficients similar to the bounds given there.

3. Starlikeness in $\Sigma_{p}$. In the following two theorems we shall obtain conditions pertaining to the starlikeness of members of $\Sigma_{p}$.

THEOREM 6. Let $f \in \Sigma_{p}, f \neq 0$, and the coefficients satisfy the condition $\sum_{n=-p+1}^{\infty}|n|\left|a_{n}\right| r^{n+p} \leqq 1, a_{0}=0$, for some $r, 0<r \leqq 1$, then $f$ is starlike for $|z|<r$.

Proof. Consider the expression

$$
H\left(f, f^{\prime}\right)=\left|z f^{\prime}+f\right|-\left|z f^{\prime}-f\right| .
$$

Replacing $f$ and $f^{\prime}$ by their series expansions we have

$$
H\left(f, f^{\prime}\right)=\left|\sum_{n=-p}^{\infty}(n+1) a_{n} z^{n}\right|-\left|\sum_{n=-p}^{\infty}(n-1) a_{n} z^{n}\right| .
$$

Hence $H\left(f, f^{\prime}\right) r^{p} \leqq-2+2 \sum_{n=-p+1}^{\infty}|n|\left|a_{n}\right| r^{n+p} \leqq 0$ which gives

$$
\left[\left(z f^{\prime} / f\right)+1\right]\left[\left(z f^{\prime} / f\right)-1\right]^{-1} \leqq 1
$$

or $\operatorname{Re}\left(z f^{\prime} / f\right) \leqq 0$ for $|z|=r$. However, this inequality holds for all circles of radius $\rho$ where $r-\delta<\rho \leqq r \leqq 1, \delta>0$, which implies that $f$ is starlike.

Suppose that $f \in \Sigma_{p}$ and $f \neq 0$. Then $g(z)=z^{p} f(z)$ is regular in $|z|<1$. For a given $\mu, \mu>0$, we can write $g(z)$ as $g(z)=[h(z)]^{2 p / \mu}$ where a particular branch has been chosen so that $h(0)=1$. Then $h(z)=1+c_{1} z+c_{2} z^{2}+\cdots$. If in the integral of Golusin mentioned in $\S 2$ we set $\lambda=\mu / p, R=\left|f\left(r e^{i \theta}\right)\right|$ we have

$$
\int_{0}^{2 \pi} R^{\lambda} d \theta=\int_{0}^{2 \pi}\left|\left[f\left(r e^{i \theta}\right)\right]^{\mu / 2 p}\right|^{2} d \theta=2 \pi\left(r^{-\mu}+\sum_{n=1}^{\infty}\left|c_{n}\right|^{2} r^{2 n-\mu}\right)
$$

which yields, upon further application of the area principle techniques, $\sum_{n=1}^{\infty}(2 n-\mu)\left|c_{n}\right|^{2} \leqq \mu$. We also note that for $z=r e^{i \theta}$ the expression $\left(z g^{\prime} / g\right)-p=\left(z f^{\prime} / f\right)$. We consider now an expression similar to (29)

$$
H\left(g, g^{\prime}, p\right)=\left|\left(\frac{z g^{\prime}}{g}-p\right)+p\right|-\left|\left(\frac{z g^{\prime}}{g}-p\right)-p\right|
$$

and note that whenever $H$ is nonpositive $\operatorname{Re}\left\{\left(z g^{\prime} / g\right)-p\right\}=\operatorname{Re}\left(z f^{\prime} / f\right)$ is nonpositive and hence $f$ is meromorphically starlike of order $p$. Therefore $f$ will be starlike whenever 


$$
\left|\sum_{n=1}^{\infty} n c_{n} z^{n}\right|-\left|\mu-\sum_{n=1}^{\infty}(n-\mu) c_{n} z^{n}\right| \leqq 0 .
$$

If $0<\mu \leqq 1$ the inequality (32) will be valid whenever $\sum_{n=1}^{\infty}(2 n-\mu)\left|c_{n}\right||z|^{n} \leqq \mu$. An application of the Schwarz inequality yields

$$
\begin{aligned}
\sum_{n=1}^{\infty}(2 n-\mu)\left|c_{n}\right||z|^{n} & \leqq\left[\sum_{n=1}^{\infty}(2 n-\mu)\left|c_{n}\right|^{2}\right]^{1 / 2}\left[\sum_{n=1}^{\infty}(2 n-\mu)|z|^{2 n}\right]^{1 / 2} \\
& \leqq \mu^{1 / 2}\left[\sum_{n=1}^{\infty}(2 n-\mu)|z|^{2 n}\right]^{1 / 2} \\
& =\mu^{1 / 2}\left[\frac{|z|^{2}}{\left(1-|z|^{2}\right)^{2}}\left(\mu|z|^{2}+2-\mu\right)\right]^{1 / 2} .
\end{aligned}
$$

The right-hand member of (33) will be less than or equal to $\mu$ provided $|z|^{2} \leqq \mu(2+\mu)^{-1}$. We easily see that the largest value of $|z|$ occurs when $\mu=1$ and hence $|z| \leqq(1 / \sqrt{ } 3)$ is the radius of starlikeness. We have the following

THEOREM 7. Let $f \in \Sigma_{p}$ and $f \neq 0$ for $|z|<1$; then $f$ is meromorphically starlike in $|z|<(\sqrt{ } 3)^{-1}$.

\section{REFERENCES}

1. M. Biernacki, Les fonctions multivalentes, Hermann, Paris, 1938.

2. J. Clunie, On meromorphic schlicht functions, J. London Math. Soc. 34 (1959), 115-116.

3. G. M. Golusin, On p-valent functions, Mat. Sb. (N. S.) 8 (1940), 277-284. (Russian with German summary)

4. A. W. Goodman, On the Schwarz-Christoffel transformation and p-valent functions, Trans. Amer. Math. Soc. 68 (1950), 204-223.

5. A. Kobori, Zur Theorie der mehrwertigen Funktionen, Japan J. Math. 19 (1947), 301-319.

6. R. Libera and M. S. Robertson, Meromorphic close-to-convex funnctions, Michigan Math. J. 8 (1961), 167-175.

7. Z. Nehari, and E. Netanyahu, Coefficients of meromorphic schlicht functions, Proc. Amer. Math. Soc. 8 (1957), 15-23.

8. C. Pommerenke, Uber einige Klassen meromorpher schlichter Funktionen, Math. Z. 78 (1962), 263-284.

9. M. S. Robertson, Multivalently starlike function, Duke Math. J. 20 (1953), 539-550.

The INSTITUTE for AdVANCEd StUdy,

Princeton, NeW Jersey

THE UNIVERSITY OF KeNTUCKY, LEXINGTON, KeNTUCKY 